\title{
La renovada importancia de los conceptos geográficos tradicionales en la construcción digital del espacio y del lugar
}

\section{Tilo Felgenhauer}

Abt. Sozialgeographie, Institut für Geographie, Friedrich-Schiller-Universität Jena, Alemania.

Recibido: 22 de diciembre de 2017. Aceptado: 29 de agosto de 2018.

\begin{abstract}
Resumen
La Geografía ha abordado la revolución digital asumiendo que la globalización implicó la pérdida de anclaje de las relaciones espaciales o, por el contrario, la persistencia de las fronteras, las desigualdades y las identidades construidas desde el lugar. Se propone aquí un modelo para analizar estos dos aspectos de manera integral. En primer lugar, se analizará el carácter y las consecuencias del cambio tecnológico y la globalización. En segundo lugar, se examinarán tres aspectos de los imaginarios geográficos tradicionales: (i) la ontología de una serie de entidades espaciales -tales como país, región, paisaje y lugar - definidas como receptáculos de características naturales y culturales; (ii) el supuesto de existencia de relaciones naturales entre el lugar y las comunidades locales, (iii) el carácter -en principio- estático de estas entidades espaciales. Las interacciones contemporáneas con los dispositivos digitales necesariamente incluyen dos aspectos: el cambio tecnológico y el pensamiento geográfico tradicional. Con la finalidad de analizar esta interacción se propone establecer una distinción entre la representación tecnológica del espacio, en términos algorítmicos, la recreación gráfica y lingüística de una geografía vivida a nivel del usuario individual y, finalmente, la referencia a topónimos a nivel de un colectivo. Los imaginarios geográficos tradicionales, entonces, serían parte integral de las prácticas digitales cotidianas de la contemporaneidad y no solo una capa residual que se opone a ellos.
\end{abstract}

PALABRAS CLAVE: TECNOLOGÍAS DIGITALES. GLOBALIZACIÓN. CONCEPTOS GEOGRÁFICOS. IMAGINARIOS GEOGRÁFICOS.

\section{The digital construction of space and place, and the renewed importance of traditional geographical concepts}

\begin{abstract}
Geography's reflection of the digital revolution either draws connections to globalization as the disembedding of spatial relations due to technological progress or emphasizes the persistence of borders, inequalities and place identities as its counterpart. The article
\end{abstract}


develops a model that treats both sides of this figure, more as intertwined than contrasting. First, an introduction is given to the character and consequences of globalization and technological change and to the traditional meanings of space and place. With regard to the latter three main aspects of traditional geographical imaginations will be outlined: (i) a physical ontology of spatial entities such as country, region, landscape or place, as clearly defined individual and distinct containers of natural and cultural features; (ii) the assumption of natural relations between place and local communities, and (iii) the (in principle) static character of those entities. Contemporary everyday interaction with digital devices necessarily reflect/include two sides: technological change and of traditional geographic thinking. In order to analyze this relationship, a distinction of numeric components, deictic language and toponymy are suggested reflecting technological system, the user individual and the community/collective. While applying this distinction, it should become clear how and why practices of using digital media and technology refer to traditional geographic concepts. Thus, traditional geographical imaginations can be considered to be an integral part of digitalized everyday practices -and do not only constitute a persistent residue opposed to them-.

KEYWORDS: DIGITAL TECHNOLOGIES. GLOBALIZATION. GEOGRAPHICAL CATEGORIES. GEOGRAPHICAL IMAGINATIONS.

PALAVRAS-CHAVE: TECNOLOGIAS DIGITAIS. GLOBALIZAÇÃO. CONCEITOS GEOGRÁFICOS. IMAGINÁRIOS GEOGRÁFICOS.

\section{Introducción: las geografías digitalizadas del mundo cotidiano, narrativas antagónicas}

El discurso geográfico contemporáneo considera a la globalización y al cambio tecnológico como las fuerzas más importantes en la transformación de las relaciones socioespaciales. La denominada revolución digital, particularmente, pareciera sustentar la visión de un poder que, con independencia de su posición geográfica, sus condiciones materiales e incluso su territorialidad política, se ha vuelto ubicuo. Se impone un cuestionamiento de las premisas geográficas tradicionales: los significados de los objetos espaciales y los principios del pensamiento espacial re-negociados. Si la innovación tecnológica promueve una expansión espacial del mundo a nuestro alcance (p. ej. Dicken, 1998) también desafía la relevancia de las categorías clásicas del pensamiento geográfico.

Por otro lado, y con el mismo grado de claridad, podemos constatar el renacimiento de límites, divisorias, desigualdades espaciales y, por consiguiente, la renovada relevancia del conocimiento geográfico y de conceptos clásicos de la geografía, tales como lugar, región, nación, territorio, distribución y difusión. Estos conceptos recobran importancia en relación a las explicaciones de un desarrollo espacial a nivel global, así como de los discursos neo-conservadores que subrayan la identidad regional y nacional. La renovada importancia de la Geografía está profundamente conectada a lo digital (p. ej. Buliung, 2011): las geografías simbólicas aparecen como un contenido de interfaz que la neogeografía (p. ej. Kingsbury y Jones, 2009; Gryl y Jekel, 2012), la geoinformación y los discursos de geolocalización social refuerzan. En efecto, las narrativas geopolíticas, los discursos sobre el renacimiento de los movimientos nacionalistas (p. ej. Edensor, 2002; Schultz, 1998) y las ideas de bio-regionalismo y consumo local (p. ej, Blake et al., 2010) cada vez ganan más atención en los medios de comunicación. La digitalización ha transformado la movilidad y los viajes; simultáneamente ha reproducido 
distintas imágenes de los lugares y de las nociones de diferencias espaciales. Todavía más: la movilidad y la aceleración de los ritmos de acción cotidiana, no solo se han incrementado, sino que también han hecho más vitales los imaginarios geográficos y han espacializado los regímenes de visión (p. ej. Lois y Hollman, 2013).

Consideramos que necesitamos modelos y teorías capaces de explicar la combinación, la superposición y la interconexión de estos imaginarios ambivalentes en nuestras prácticas cotidianas, en lugar de discutir si la globalización o la regionalización constituyen la fuerza dominante, o de evaluar de manera conclusiva el peso de cada uno de sus posibles escenarios. Por ejemplo, sin estar atravesados por una sensación disruptiva o ambivalente, cada vez que utilizamos las redes globalizadas de comunicación digital colocamos la dirección de un lugar. Con el propósito de contar con un marco analítico para explicar la multiplicidad de estos fenómenos, este artículo introduce un modelo básico y descriptivo de las geografías del uso cotidiano de las tecnologías, especialmente de la semántica espacial que se pone en juego en la interacción con dispositivos tecnológicos. Se trata de un abordaje que busca responder a qué conceptos de espacio y lugar se evocan al interactuar con los sistemas de tecnología digital y sus infraestructuras.

Establecer un marco teórico para el análisis de la interacción tecnología-usuario nos permitirá construir una explicación teórica para entender por qué nuestros imaginarios geográficos se mantienen asombrosamente inalterados, a pesar de que las tecnologías digitales aumentan de modo constante nuestras capacidades de actuar atravesando distancias y de comunicarnos prácticamente con cualquier lugar (y en cualquier momento). El artículo desarrolla esta argumentación en cuatro partes. En la primera, se discuten los modelos generales sobre las tecnologías y los cambios socio-espaciales. En la segunda, se presenta brevemente la narrativa opuesta de pensamiento geográfico tradicional. La tercera sección introduce un modelo de interacción que se deriva de las teorías de las tecnologías y fenomenología social, como una metodología de aproximación al nivel de las prácticas cotidianas. Finalmente, se exponen las contribuciones de este abordaje para entender el renacimiento de imaginarios geográficos tradicionales en un contexto de innovación digital.

\section{La expansión de los sentidos y el estrechamiento de los mundos: los abordajes de la tecnología, la modernidad y la globalización}

La Antropología ha brindado un modelo bastante convincente sobre la relación entre el hombre, la naturaleza y la tecnología. El espíritu de invención ha compensado las limitadas habilidades del hombre como especie biológica (como la falta de instintos y de un hábitat natural particular) (Herder 2001 [1772]; Gehlen 1958). Así, por ejemplo, el martillo extiende y fortalece la mano humana, en tanto que el telescopio amplifica las habilidades del ojo humano. La tecnología incluso ofrece sustitutos ante la falta de determinados órganos como las branquias o las alas (Gehlen 1958; 2007 [1957]). Desde este modelo el hombre se encuentra forzado a crear los medios e instrumentos -tecnologías- que funcionan como una extensión de su cuerpo y sus sentidos, y simultáneamente cuenta con las posibilidades para hacerlo. Esta forma de entender la tecnología ha influenciado la teoría de los medios de comunicación. Marshall McLuhan 
y Derrick de Kerckhove, los teóricos clásicos de los medios de comunicación, imaginaron los medios electrónicos como extensiones de los sentidos de los hombres: el hombre actúa en tiempo real atravesando las distancias y se convierte en parte de una aldea global (McLuhan, 1964). Los teóricos del siglo XXI continúan refiriéndose en el marco de esta idea clásica. Steve Mann sostiene que "la computadora personal comienza a funcionar como la extensión de la mente y del cuerpo" (Mann 2001, 7; citado por Thrift (2004a, 183). ${ }^{1}$

Esta concepción de la tecnología también subyace en los modelos de representación espacial de los cambios socio-tecnológicos y de la globalización. La tecnología es considerada la fuerza motriz que permite atravesar distancias espaciales, borrar las limitaciones materiales y correr las fronteras del hombre y de la sociedad (Véase Dicken 1998: 152; Thrift, 1996: 42; con referencia a Bradley 1988: 2). Dicken (1998: 152; con referencia a McHale) ilustra la correspondencia existente entre las revoluciones tecnológicas en el transporte y los distintos niveles de comprensión del mundo. Así, en el curso de la historia de la modernidad -desde los carruajes tirados por caballos hasta los aviones- los medios de transporte vienen acortando las distancias: gráficamente este proceso podría presentarse con un globo terráqueo que se va haciendo cada vez más pequeño hasta convertirse, finalmente, en un pequeño punto. Thrift (1996: 42; con referencia a Bradley, 1988: 2) también advierte este proceso valiéndose de una imagen que presenta una comparación de las distancias que cruzan a lo largo de su vida individuos de generaciones diferentes: mientras que el bisabuelo recorre distancias limitadas a su región de origen, el abuelo visita la capital, el padre ya tiene familiaridad con el continente y el hijo, potencialmente, recorre todo el mundo. Además, el cambio tecnológico implica una sincronía con la percepción individual y social de dicha transformación, como sostiene Thomson: ${ }^{2}$ "El desarrollo de los nuevos medios de comunicación y de transporte también afecta las experiencias espaciales y temporales características de la vida en sociedad" (1995: 33).

Los lugares de todos los continentes ya no solo están potencialmente al alcance, sino que también, la intensidad de las relaciones entre ellos crece de modo constante en la era de la información. Este fenómeno se visualiza en la densidad que toman las líneas existentes entre diferentes puntos en el globo terrestre en ciertas imágenes debido a la existencia de conexiones más estrechas entre los diferentes lugares de la superficie. ${ }^{3}$ Este tipo de cartografías sugiere que la globalización ha alcanzado su máxima expresión.

También se ha sostenido que esta expansión espacial de la movilidad y las comunicaciones se correspondería con la difusión de los derechos humanos y la integración de carácter cosmopolita. El colectivo social en el cual nos entendemos se ha expandido desde un círculo inmediato (familia, tribu) a la nación y, todavía más, a la humanidad como un colectivo global. ${ }^{4}$ En este sentido, Pinker y Goldstein (2012) afirman que la historia mundial y la globalización conducen al establecimiento de una moral universal

1 Donna Harraway (2013), de modo implícito, se refiere a este modelo al introducir la categoría de cyborg como un híbrido que no solo externaliza, sino que también internaliza, los elementos tecnológicos.

2 Este supuesto, aunque posible en una perspectiva histórica de larga duración, no debería aplicarse tan rápidamente a la tecnología digital.

3 El conocido término espacio de flujos, propuesto por Manuel Castells, representa un imaginario similar.

4 Una tesis que se conecta con la controvertida idea de "ciudadanía global" (ver Lough y Moore McBride, 2014: 458). 
y una empatía colectiva global. En relación al telos de la globalización, los abordajes radicales incluso sugieren que, asumiendo que el espacio es un marco fijo de la acción social y del desarrollo tecnológico, se ha borrado la relevancia de la dimensión espacial (Virilio 1999; Cairncross 1997). La superficie terrestre sería un telón de fondo estático ante el cual la aceleración del movimiento de los objetos, las personas y la información pareciera resultar todavía más sorprendente.

Barry Wellman (2002) ha delineado una perspectiva muy diferente sobre el impacto de la globalización compartiendo el supuesto de la expansión espacial de las relaciones sociales. Las tecnologías promueven una interacción persona a persona, en lugar de una comunicación puerta a puerta y lugar a lugar. Sin embargo, lejos de interpretar la extensión espacial de las relaciones sociales como la emergencia de un colectivo universal, Wellman sugiere la emergencia de un individualismo en red como el resultado de la comunicación global digital (Wellman, 2002: 10; ver también Miller 2011: 225). La interacción individual, con un acceso limitado a los desconocidos, balancea la promoción de grandes comunidades y sus identidades.

Los abordajes más radicales sugieren la desaparición de la importancia de la dimensión espacial. Paul Virilio describe un período de aceleración y desaparición del espacio y del tiempo que resulta en un "desierto del tiempo-mundo" (Virilio, 1999, citado en Mersch, 2006: 170). Trevor Barnes apunta de un modo crítico que el concepto de "globalización no es sobre el mundo, sino sobre su desaparición. [...] El problema con la globalización es que el mundo desaparece; un mundo en el cual no existiría más el dónde" 5 (Barnes, 1998: 331). Cairncross aboga por la muerte de la distancia debido a la introducción de las nuevas tecnologías de la comunicación en las prácticas económicas.

Con frecuencia estas posiciones han provocado fuertes críticas tanto desde un ángulo empírico como teórico. Uno de los argumentos empíricos que cuestiona la idea de un mundo aplanado apunta a que este estadio en la historia no es tan novedoso como suele presentarse. La distribución espacial de las tecnologías todavía constituye una brecha digital. Y desde la esfera social, se evidencia que el renovado interés individual y político en las identidades regionales emerge precisamente (y no a pesar) de la intensificación de la comunicación global. La glocalización (Robertson, 1995) es probablemente el término más conocido para identificar este proceso. Paasi (2003), Bromley (2010), Wodak et al. (2009), y muchos otros, discuten este asunto en profundidad. Pero también hay otro problema interno de ambivalencia en casi todas las teorías que se han introducido aquí, que tiene como causa un principio de representación subyacente en todos estos abordajes: de modo implícito todos ellos se refieren a una concepción tradicional del espacio, a pesar de que trabajan con metáforas y vívidas visualizaciones cartográficas de los cambios históricos y espaciales.

Estos abordajes suponen que el espacio es un marco fijo tanto de la acción social como del desarrollo tecnológico. De hecho, resulta todavía más sorprendente que la superficie terrestre sea concebida como un telón estático ante la aceleración del movimiento de flujos de personas e información. Básicamente, constituye la mirada de un observador externo en sintonía con una perspectiva convencional de la cartografía que, simultáneamente, carga los beneficios y las limitaciones de la visión a distancia: ofrece nuevas 
perspectivas que, al mismo tiempo, promueven la idea de un espacio extraño/ ajeno a las experiencias cotidianas. La concepción del globo terrestre como un espacio estático se aplica para sustentar las declaraciones de transformaciones espaciales innovadoras.

Si estas visiones esquemáticas del desarrollo socio-espacial han devenido en sentido común, también es cierto que han dejado abierto un interrogante: ¿Cómo relacionar estos macro-modelos con una micro escala, la escala de las acciones cotidianas? Todavía más: estos modelos asumen un espacio absoluto, concepción que no puede articularse a una geografía de lo cotidiano (Buttimer, 1976), a una escala micro o al campo del uso de las tecnologías. Para abordar estos aspectos debemos apelar a la fenomenología, a la teoría-acción y al pensamiento contemporáneo de la geografía cultural.

\section{Orígenes y estructura de la persistencia de imaginación geográfica tradicional}

Deberíamos considerar otra línea de análisis, otra narrativa sobre la historia del espacio y del lugar bien diferente e incluso, opuesta a la que se ha delineado previamente. Esta narrativa se origina en la fase formativa de la Geografía como disciplina aunque, como intentaré demostrar, todavía forma parte (y probablemente con una fuerza creciente) de las prácticas y conocimientos cotidianos. Podríamos denominarlos "imaginarios geográficos tradicionales" con referencia a la reconstrucción crítica de la historia de la Geografía de Livingstone (2008: 260) o a la teoría constructivista de los sujetos del pensamiento geográfico de Derek Gregory en su libro Geographical Imaginations. Esta narrativa identifica un conjunto de supuestos y conceptos que son constitutivos del pensamiento geográfico tradicional o clásico. Analizaremos tres de ellos en contrapunto a la narrativa de la globalización y a las ideas de desaparición del espacio.

\section{Entidades espaciales como contenedores de objetos}

El primer elemento de esta narrativa tradicional se refiere a la identificación y definición de unidades espaciales como contenedores de elementos naturales y culturales. Entidades como lugares, paisaje, región, país emergieron como objetos genuinos y casi exclusivos de la investigación geográfica. Luego se volvieron eficaces para la institucionalización de la geografía y su legitimación como una disciplina científica. El carácter natural de los paisajes y la idea de límites o bordes naturales se fijó como un hecho ampliamente aceptado. Los límites, considerados objetivamente observables, entonces fueron identificados por la geografía, no solo como parte de una exploración científica, sino como parte de una acción política y de desarrollo cultural. Dos ejemplos del discurso alemán pueden ilustrar esta idea básica. ${ }^{6}$ Un texto muy utilizado a fines del siglo XVIII señalaba: "Los límites de los países están determinados de modo predominante por la naturaleza, por sus montañas, mares y ríos". ${ }^{7}$ Posteriormente, el geógrafo alemán Alfred Hettner -conocido por el concepto de länderkunde, frecuentemente traducido como geografía regional- asumía un orden espacial natural en la territorialización

6 Me referiré a la literatura alemana, aunque existen muchos paralelos y discursos interconectados particularmente con el mundo anglosajón.

7 “Die Grenzen der Länder sind mehrenteils von der Natur bestimmt, durch Gebirge, Meere und Ströme...” (Gaspari 1799: 66; citado en Eisel y Schultz 2008: 76) 
política. Hettner aceptaba que "en la mayoría de los casos, los territorios estatales se corresponden con divisiones naturales". ${ }^{8}$

\section{El carácter estático y estable de las entidades espaciales}

En general, la estabilidad de las entidades espaciales (regiones, paisajes, países) se aceptaba como un orden armónico representado en un mosaico de lugares. También, que este orden natural y pre-fijado tenía su origen en fuerzas divinas que distribuían las cosas en su lugar apropiado. Como sostenía la obra de Carl Ritter a principios del siglo XIX: "resulta claro que con la finalidad de evitar la desaparición cada comunidad humana y cada persona debe ser consciente de los poderes internos y externos, de los poderes de sus vecinos y de las condiciones que los pueden afectar desde el exterior". 9

Desde esta perspectiva, las transformaciones y los conflictos se presentan como estados que las entidades espaciales necesariamente deben atravesar. Todavía presente en la percepción pública del orden mundial, esta perspectiva queda particularmente evidenciada en la atribución a una incorrecta interpretación de las condiciones naturales como explicación ante la declinación de determinados regímenes o culturas.

\section{La estructura de las entidades espaciales como complejos naturales- culturales}

Una vez aceptada la idea de las unidades espaciales como contenedores, también queda establecida una relación similar entre la naturaleza y la cultura. En esta perspectiva se asume que existen relaciones naturales entre el lugar y sus habitantes. En tanto la comunidad y la cultura se definen en función de su extensión y localización, la Geografía pasa a ser el campo de conocimiento experto para estudiarlo (y no la Sociología o las ciencias culturales). Desde una perspectiva crítica se ha denominado geo-determinismo a este modo de pensamiento que asume una relación unidireccional entre el lugar y la cultura: la naturaleza determina el desarrollo cultural, así como establece límites al potencial económico de un lugar particular. El poder de las condiciones naturales determina tanto el carácter cultural como el potencial de desarrollo. Por ejemplo, a principios del siglo XX Ellen Semple en el libro Influences of Geographic environment (1911: 1) señalaba:

El hombre es producto de las condiciones de la superficie terrestre. Esto significa que no solo es un hijo de la Tierra, sino que la Tierra lo ha alimentado y criado, ha dirigido sus pensamientos y confrontado con dificultades que han fortalecido su cuerpo y refinado sus saberes. En las montañas, le ha dado músculos de hierro en sus piernas para ascender las pendientes; en las costas esos músculos más débiles se contrarrestan con el gran desarrollo pectoral y de sus brazos para remar.

8 (dass) "die meisten Staaten im großen und ganzen mit natürlichen Abteilungen zusammenfallen" (Hettner 1907c; citado en Eisel y Schultz 2008: 86).

9 so ist es klar, dass auch jeder menschliche Verein, jedes Volk seiner eignen innern und äußern Kräfte, wie derjenigen der Nachbarn, und seiner Stellung zu allen von außen hereinwirkenden Verhältnissen inne werden sollte, um sein wahres Ziel nicht zu verfehlen" (Ritter 1822: 1) 
La dinámica del proyecto colonial de expansión en la frontera de América del Norte, a fines del siglo XIX, estuvo sujeta al pensamiento geo-determinista en los márgenes de la Geografía, como lo muestra la idea de Frederick Jackson Turner (1996: 4), al referirse a la transformación cultural determinada por los movimientos espaciales:

La naturaleza domina el colono. Lo encuentra con su vestimenta, herramientas, modos de transporte y pensamiento europeos. Lo saca del camino del auto y lo coloca en una canoa de madera. Le retira el ropaje de la civilización [...] el avance de la frontera supone un escape de la influencia de Europa...

El pensamiento geo-determinista no es solo un fenómeno del pasado; permanece y todavía configura discursos públicos y el sentido común respecto al espacio y a la cultura. En efecto, el reciente éxito editorial Prisioners of Geography de Tim Marshall proclama que las sociedades son prisioneras de la Geografía, como el propio título del libro lo enuncia: "El lugar donde vivimos nos modela. Modela las guerras, el poder, la política, el desarrollo social. Las características geográficas son los factores dominantes en la determinación de lo que la gente puede o no puede hacer" (Marshall, 2014: 1).

El geo-determinismo fue criticado e incluso invertido al asignar el rol dominante a la cultura en lugar de la naturaleza en el pensamiento de Paul Vidal de la Blache y de Carl Sauer: "El paisaje natural es sujeto de la transformación de las manos del hombre, su [...] factor morfológico más importante. A través de su cultura utiliza las formas naturales, las modifica y en algunos casos las destruye" (Sauer, 1963 [1925]: 341). Pero incluso con esta inversión explícita en la lógica de la relación entre el hombre y la naturaleza, las entidades de la superficie terrestre, objeto de la Geografía, se mantienen intactas. Mientras que la teoría geográfica ha cuestionado cada vez más estos tres conceptos básicos, por ejemplo, a través del análisis espacial cuantitativo o de la aplicación de la perspectiva hermenéutica, el discurso público sigue aferrándose a estas ideas. Es por este motivo que, en tanto corpus de conocimiento que todavía continúa otorgando sentidos por fuera de la Geografía, debería formar parte de la agenda de investigación de la geografía, ya no como una serie de supuestos, sino como objetos de indagación. Estas ideas se consolidaron como sentido común de las sociedades modernas, a través de los sistemas educativos y de los medios de comunicación, precisamente cuando la evidencia empírica comenzó a ser desafiada por la globalización.

El antagonismo/oposición de las dos narrativas -el poder de transformación de la narrativa de la globalización y la persistencia de los conceptos de la tradición geográfica- es uno de los asuntos claves que influencia nuestros usos diarios de la tecnología digital (Cuadro 1). 
La renovada importancia de los conceptos...

TILO FELgenHAUER

Cuadro 1. Contrapuntos de la narrativa de la globalización y de las ideas básicas de la tradición geográfica en relación a la tecnología, el espacio y la sociedad. Fuente: Elaboración propia.

\begin{tabular}{lcc}
\hline Rol de la tecnología & Teoría de la globalización & Tradición geográfica \\
\hline Concepto de espacio & $\begin{array}{c}\text { Superficie de contacto } \\
\text { inmediato. Intercambio y } \\
\text { movilidad ilimitada. }\end{array}$ & $\begin{array}{c}\text { Contenedor de la naturaleza/ } \\
\text { cultura. Límites precisos y } \\
\text { estáticos. }\end{array}$ \\
\hline Entidad social dominante & sociedad cosmopolita & $\begin{array}{c}\text { comunidad definida } \\
\text { localmente }\end{array}$ \\
\hline $\begin{array}{l}\text { Idea dominante de progreso y } \\
\text { teleología }\end{array}$ & $\begin{array}{c}\text { Sociedad global; } \\
\text { Homogeneización; telos } \\
\text { Universalismo }\end{array}$ & $\begin{array}{c}\text { Armonía, estabilidad en el } \\
\text { descubrimiento/ Límites } \\
\text { naturales pre-determinados/ } \\
\text { Armonía= separación. }\end{array}$ \\
\hline
\end{tabular}

La próxima sección expone un marco analítico que busca entender la semántica de la acción con las tecnologías digitales y, como segundo paso, explicar la persistencia de la tradición geográfica en los ambientes digitales.

\section{La interacción con la tecnología digital como una práctica cotidiana}

Podríamos tomar elementos de cada uno de los giros que ha experimentado la disciplina en las últimas décadas para analizar cómo son evocadas, reproducidas y transformadas las geografías del uso de la tecnología en la escala micro. Tal vez el movimiento que podríamos tomar como paraguas sería el giro práctico que atraviesa todos los giros, entre ellos el cualitativo, el lingüístico, el material y el performativo. En un sentido amplio, centrarnos en las prácticas podría incluir la investigación sobre la vida del individuo, sus experiencias, sus rutinas, su creatividad en el reconocimiento y solución de problemas cotidianos, sus interpretaciones sobre el mundo material y las relaciones sociales. Incluso podríamos entender que la mayor atención otorgada a la emoción es parte de este giro práctico. En definitiva, una geografía de las prácticas considera la constante producción de sentido tanto a nivel material como simbólico y destaca las habilidades y la creatividad de las personas, así como el conocimiento incorporado en ese entramado de relaciones socio-tecnológicas (de Certeau, 1984).

Este sentido más laxo del giro práctico en relación a la tecnología digital sugiere varias contribuciones desde la geografía humana. Como ha señalado Stephen Graham en torno a las implicancias de las tecnologías digitales en la economía política (1998, 2005), las "geografías diseñadas por los software" (Graham, 2005) se erigen como una nueva cualidad en la codificación digital de la vida urbana, las relaciones económicas, sociales y del ejercicio del poder político. Con el término "prácticas transductivas" Dodge y Kitchin $(2005,2011)$ destacan que las tecnologías digitales (código) son parte de un complejo de prácticas y operaciones por momentos intencionales, pero en la mayoría de los casos automatizadas, a veces visibles, pero generalmente ocultas. Entonces, estas prácticas simbólicas que invocan espacios y lugares deberían ser identificadas y teorizadas. Para abordar las espacialidades producidas por estas tecnologías, estos autores proponen distinguir los "objetos codificados" - mercancías con códigos de barra-, los "espacios 
codificados" -como la casa, el supermercado- y los "espacios-código" -lugares cuya existencia y funcionamiento dependen de un código, como los aeropuertos (Dodge y Kitchin, 2005: 172)-.

La fenomenología ofrece una vía fructífera para el análisis de esta cuestión partiendo de la observación de lo banal (Berger y Luckmann, 1966). Desde esta perspectiva, los usuarios legos utilizan la tecnología de manera rutinaria, con mayor o menor integración en sus acciones y a través de interfaces que regulan la interacción con los aparatos tecnológicos y las infraestructuras. La ubicuidad de las infraestructuras digitales las torna simultáneamente presentes e invisibles en la vida cotidiana: están totalmente integradas en muchas de nuestras acciones incluso sin que lo percibamos o comprendamos como usuarios. Según el filósofo Hans Blumenberg (1981) esta creciente brecha entre lo performático (Leistung) y su transparencia (Einsicht) es precisamente el resultado del progreso tecnológico. Así, las complejas operaciones que están detrás de cada interfaz se convierten en una caja negra: los usuarios utilizan los sistemas como resultado de una rutina sin que esto implique que conozcan las operaciones internas del sistema. La cesión de esta carga de trabajo de la tecnología conduce a la alienación tanto de los usuarios, de los expertos en la tecnología y de las relaciones espaciales que emergen por detrás de cada interfaz. La especialización del conocimiento, y de otros roles y tareas, promueve y requiere relaciones de confianza en sistemas abstractos. En efecto, este crecimiento enorme de la productividad y del potencial tecnológico se basa en delegar en otros (por ejemplo, los especialistas que han inventado nuevos lenguajes como el código), en confiar en nuevas formas de pensamiento (como la ciencia) y en innovaciones tecnológicas opacas pero útiles en nuestra vida cotidiana. Lejos de ser un escenario excepcional, como sostiene Anthony Giddens (1990), esto es una consecuencia propia de la modernidad.

Podríamos derivar tres elementos básicos de interacción, cada uno de ellos con su propia semántica espacial, a partir este dualismo clásico de dos mundos altamente diferenciados: por un lado, los sistemas expertos y por otro, los usuarios y su experiencia cotidiana:

» la representación tecnológica del espacio en términos algorítmicos y numéricos (y el correspondiente esfuerzo de los usuarios por comprenderlos);

» la emulación o recreación gráfica y lingüística de la geografía vivida que permite a los usuarios interactuar de modo intuitivo con el sistema, a través de interfaces sensibles a la localización;

" las referencias a construcciones complejas meta-contextuales y meta-individuales como la región y la nación.

Como resultado pueden distinguirse tres modos de interacción imbricados con tres espacialidades diferentes (Cuadro 2): el algorítmico/numérico, el egocéntrico y el toponímico. Los próximos párrafos desarrollan este esquema en detalle. 
TiLo FeLgenHAUER

Cuadro 2. Modos de interacción y su correspondiente espacialidad. Fuente: Elaboración propia.

\begin{tabular}{|c|c|c|c|}
\hline $\begin{array}{c}\text { Entidad dominante } \\
\text { en el contenido de la } \\
\text { interacción }\end{array}$ & Modo de interacción & $\begin{array}{c}\text { Forma de } \\
\text { espacialidad }\end{array}$ & $\begin{array}{c}\text { Correspondencia } \\
\text { con los paradigmas } \\
\text { geográficos } \\
\end{array}$ \\
\hline Sistema & $\begin{array}{c}\text { Lectura y escritura del } \\
\text { lenguaje algorítmico } \\
\text { y numérico }\end{array}$ & $\begin{array}{l}\text { Numérico. Código } \\
\text { numérico de los } \\
\text { lugares y las } \\
\text { relaciones espaciales } \\
\text { (Códigos postales, } \\
\text { Dirección IP) }\end{array}$ & $\begin{array}{l}\text { Análisis espacial, } \\
\text { geografía } \\
\text { cuantitativa. }\end{array}$ \\
\hline Usuario (individual) & $\begin{array}{l}\text { Lenguaje de la } \\
\text { interfaz, selección } \\
\text { y edición de datos } \\
\text { en función de } \\
\text { la experiencia } \\
\text { individual. }\end{array}$ & $\begin{array}{c}\text { Expresiones deícticas } \\
\text { (punto de referencia) } \\
\text { y gráficos que } \\
\text { emulan la percepción } \\
\text { de las realidades } \\
\text { espaciales, diseño } \\
\text { de interfaces } \\
\text { egocéntricas } \\
\text { (representación } \\
\text { espacial de las } \\
\text { áreas circundantes } \\
\text { en juegos o } \\
\text { aplicaciones) }\end{array}$ & $\begin{array}{l}\text { Geografía } \\
\text { Humanística }\end{array}$ \\
\hline Comunidad & $\begin{array}{l}\text { Lenguaje de la } \\
\text { interfaz; introducción } \\
\text { del lenguaje de la } \\
\text { vida cotidiana en el } \\
\text { diseño de la interfaz. }\end{array}$ & $\begin{array}{l}\text { Topónimos. } \\
\text { Localización de } \\
\text { lugares y espacios } \\
\text { a través del uso de } \\
\text { topónimos (nombres } \\
\text { de ciudades, países). }\end{array}$ & $\begin{array}{l}\text { Geografía Regional: } \\
\text { Länderkunde }\end{array}$ \\
\hline
\end{tabular}

\section{El sistema tecnológico de representación del espacio en términos algorítmicos y numéricos}

Las interfaces gráficas y pantallas táctiles contemporáneas fueron precedidas por un período en el cual el uso de los instrumentos digitales necesariamente requería conocer y manejar el código (Hartmann, 2012; Hellige, 2008). Esta interacción estaba diseñada más en función de los requerimientos inherentes al sistema que en función de los conocimientos de los usuarios legos.

En nuestra vida cotidiana todavía es posible encontrar vestigios de aquella etapa, especialmente en la organización espacial de las tecnologías de la comunicación. La representación numérica del lugar y del espacio es uno de los indicadores más emblemáticos: en algunos casos todavía nos vemos obligados a utilizar códigos postales, también tenemos códigos de acceso que aluden a lugares en las redes de comunicación telefónica y, otras veces, tenemos que lidiar con una dirección de IP (IP- address) para poder acceder a una red de internet. Un ejemplo particular de esta apropiación del código geo-digital se da en las experiencias denominadas Geografías participativas o colaborativas (Goodchild, 2007). El término alude precisamente al hecho de que el uso de la geoinformación ya no se restringe a usuarios expertos o profesionales. Por el contrario, los denominados "citizen-sensors" (Goodchild, 2007) o "prosumidores"10 
no sólo utilizan de modo innovador y creativo los sistemas de información geográfica sino que también los aplican para usos individuales (Sui, 2004; Elwood et al. 2011; Bittner et al.. 2013). También crean sus propias plataformas y aplicaciones a partir de otras como Open Street Map (Perkins, 2014).

El creciente acceso a estas tecnologías podría conducir a un mejor entendimiento de la información geográfica en el marco de una mayor participación ciudadana a nivel local y global en diversos campos como la política, el planeamiento y la educación espacial. Desde esta perspectiva, el manejo de los sistemas de información geográfica podría estimular una mayor democratización, con una mayor participación cívica en la política global y local, en los procesos de planificación y en la educación espacial. Sin embargo, no deberíamos olvidar que solo un número reducido de usuarios maneja los algoritmos y el lenguaje de los sistemas de geoinformación. La interacción con el código es una excepción a la regla.

\section{La recreación gráfica y lingüística de la geografía del usuario}

Actualmente es mucho más frecuente que las interacciones con la tecnología digital se den con interfaces gráficas y portátiles que no exigen del usuario el conocimiento de los algoritmos, el manejo del código de operación o el conocimiento de las representaciones numéricas de las entidades espaciales. Las interfaces recrean el espacio cotidiano del usuario individual: un mundo que tiene al usuario como centro a partir del cual se va desplegando una serie de capas concéntricas que indican menor relación hacia la periferia (Schütz y Luckmann, 2003; Bollnow, 2010 [1963]). En virtud de su referencia al individuo suelen categorizarse como interfaces egocéntricas. La navegación satelital es el caso más típico de esta doble faz: una complejidad que no se ve y un despliegue de posibilidades digitales que emulan la percepción de cada individuo. La cartografía tradicional, construida en torno a la idea de la visión de totalidad y de una perspectiva universal, es reemplazada por la constante adaptación y selección de síntesis gráficas de la información. ${ }^{11}$ Los locative media (la navegación satelital y las aplicaciones de realidad aumentada) son representativos de este cambio.

Desde una perspectiva semiótica, se observa un pasaje desde signos algorítmicos abstractos (y arbitrarios) a icónicos, es decir, con interfaces y diseños gráficos que emulan las espacialidades del mundo real; la apariencia abstracta cede ante una apariencia más cercana y semejante a la experiencia. En términos lingüísticos podría describirse como un salto desde un vocabulario arbitrario (por ejemplo, con la nominación de los lugares a través de un lenguaje experto) a expresiones deícticas y situacionales tales como izquierda, derecha, aquí, allá que dependen de la situación contextual del cuerpo del individuo en cuestión -similares a la comunicación cara a cara (ver Berger y Luckmann, 1966: 28; Thompson, 1995: 82)-. Por ejemplo, la práctica de señalar objetos guía y modelar la visión del usuario, rutinas que por otra parte están cada vez mejor integradas en los diseños de las interfaces contemporáneas.

11 Según Latour y November (2010) transitamos un pasaje desde mapas miméticos a mapas navegacionales. Desde nuestro punto de vista, el carácter mimético se mantiene en los mapas navegacionales. Lo que cambia es el objeto de la mímesis: ya no es la superficie terrestre, sino la percepción espacial del usuario. 
Esta convergencia de la tecnología y la geografía del usuario se refleja de manera más o menos explícita en las discusiones de las geografías corporales, del afecto y de la emoción (Thrift, 2004b; Kwan, 2007; Simpson, 2014; Ash y Simpson, 2014). Las otrora denominadas realidades virtuales implican un abordaje desde la perspectiva del usuario que se distancia de las aproximaciones que privilegiaban la perspectiva estructural (Shaw y Warf, 2009; Günzel, 2010; Abend, 2013). El objetivo de estos trabajos es escudriñar las experiencias cotidianas centrándose en los espacios (y lugares) que emulan las tecnologías digitales, dimensión que se presenta en la categoría usuario-individual del esquema del Cuadro 2.

\section{Toponimia compartida en el lenguaje interactivo}

Además de las capas de lenguaje de código y deíctico, a las cuales ya nos hemos referido, se agrega otra capa más: el vocabulario coloquial del espacio compartido por una comunidad lingüística y social. Por ejemplo, el significado de la toponimia (nombres de calles, ciudades, regiones, Estados-nación) forma parte del conocimiento más básico y cotidiano. Los términos de estas entidades espaciales constituyen simultáneamente un lenguaje experto y un lenguaje espacial del sentido común, un conocimiento compartido que brinda marcos de referencia a las prácticas de interacción con los sistemas tecnológicos. Son comunes en el sentido meta-contextual. Los topónimos no requieren un conocimiento tecnológico ni subjetivo pues ya forman parte de la imaginación espacial de las comunidades digitales como una síntesis de las redes sociales y de los locative media. Pero también forman parte del conocimiento relativo a la tradición geográfica. Estas plataformas hacen referencia a nombres comunes de lugares con la finalidad de promover la participación y compartir un conocimiento estructurado espacialmente. De este modo, el imaginario geográfico compartido por una comunidad y representado por simples topónimos -ya sea del barrio, la ciudad, la región o la nación - toma mayor importancia con las nuevas tecnologías tanto en el diseño de las redes sociales y de las interfaces como en los locative media. Por ello, las investigaciones sobre la construcción del lugar, de lo regional (Bialasiewicz, 2003; Paasi, 2003), de la identidad nacional (Edensor, 2002; Wodak et al. 2009) y del rol de los medios de comunicación en la formación de la identidad (Felgenhauer et al. 2005; Bromley, 2010) tendrían que revisarse e incluir los medios digitales.

Las tecnologías digitales están reproduciendo de manera creciente las convenciones geográficas que ya son familiares a nuestro imaginario geográfico. En primer lugar, la emergencia de una geografía que se centra en el entorno del cuerpo del individuo (tercera línea del Cuadro 2). En segundo lugar, hay un resurgimiento en la importancia de la toponimia de los lugares (cuarta línea del Cuadro 2). Las tecnologías emulan de modo creciente los imaginarios geográficos relacionados a la percepción corporal o a las categorías clásicas de la tradición geográfica como el lugar, la región o la nación. La espacialidad, por lo general, se presenta de una manera conocida/familiar.

\section{La interacción con el lenguaje espacial y los paradigmas del pensamiento geográfico}

El esquema presentado en el Cuadro 2 podría contribuir a la discusión de las conexiones entre el trabajo científico en torno a los conceptos de espacio y lugar, y la construcción cotidiana del espacio y del lugar en un sentido de doble hermenéutica. ${ }^{12}$ En este sentido,

12 Anthony Giddens (1984: 374) acuñó la frase “doble hermenéutica” para referirse al feedback del conocimiento 
las categorías delineadas en el marco analítico podrían ser leídas como reflexiones sobre los paradigmas geográficos ya establecidos. Mientras que las geografías algorítmicas podrían albergar conceptos y principios similares a las del análisis espacial, las geografías deícticas del usuario individual podrían conectarse a las ideas de la Geografía Humanística, y la tercera categoría (topónimos compartidos por un colectivo) podría corresponderse con una geografía de matriz sociológica que se centra en la formación de identidades locales, regionales y nacionales. En este sentido, las hipótesis aquí presentadas no solo se refieren a la persistencia y cambio de los imaginarios geográficos tradicionales, sino que provocan interrogantes sobre las similitudes conceptuales entre el conocimiento científico y las geografías cotidianas. El modelo analítico podría servir entonces como herramienta heurística para la investigación empírica pero también, en un sentido amplio, podría constituir un itinerario para interrogarnos sobre las relaciones entre la teoría geográfica y la estructura semántica de las geografías cotidianas.

\section{La interacción con las tecnologías digitales y el renacimiento de conceptos geográficos tradicionales}

Resulta interesante observar que en un contexto de innovaciones digitales (que permiten la comunicación a distancia, la movilidad virtual y el continuo intercambio personal) la localización y la distancia espacial todavía marcan diferencias, incluso el sentido de pertenencia a un lugar es cada vez más común. Cabría preguntar por qué todavía vivimos en un mundo en el que los imaginarios geográficos tradicionales siguen resultando tan familiares. Para responder a esta pregunta, el modelo de interacción ofrece una hipótesis explicativa. La primera parte de esta hipótesis es de naturaleza negativa: es una explicación sobre la ausencia de nuevos imaginarios geográficos en estos ambientes digitales. La segunda parte, es un argumento positivo en torno a la renovada presencia de los imaginarios geográficos tradicionales en estas prácticas.

\section{El carácter opaco de las geografías de la infraestructura digital}

El concepto de interacción que presentamos tiene dos aristas de polarización. Por un lado, expertos y sistemas expertos; por otro lado, los usuarios y sus vidas cotidianas. La hipótesis central es que la grieta existente entre el conocimiento experto y el conocimiento de los no especialistas evita que hagamos la experiencia de las operaciones que son inherentes a las tecnologías e infraestructuras digitales. Dicho de otro modo, las redes de comunicación, así como otros contextos de desarrollo tecnológico se caracterizan por su opacidad. El usuario típico tiene sus interacciones limitadas a la interfaz y desconoce las operaciones técnicas que ocurren en esa caja negra. Así, las espacialidades inherentes a las tecnologías digitales quedan desconectadas de la interacción con los instrumentos y sus interfaces y, en la mayor parte de los casos, los usuarios no son capaces (o ni siquiera lo desean) de entender las geografías de las tecnologías digitales en la materialización de su infraestructura. El código se presenta como algo críptico y difuso que los usuarios no pueden definir con claridad y, como consecuencia, se inclinan a conceptos espaciales más intuitivos. Hasta los ensayos de representarlas con nuevas metáforas espaciales se caracterizan por ser bastante imprecisos -por ejemplo,

científico social en lo cotidiano. En este sentido, el conocimiento científico moldea el abordaje inicial del proyecto de investigación. 
la nube-. Por ello, la compleja espacialidad de las tecnologías digitales queda detrás del sistema y se orienta a espacialidades centradas en el usuario y espacialidades de toponimia compartida. Esta hipótesis se aparta de la noción de prosumidores (Toffler), usuarios que comprenden el código y diseñan la espacialidad de su vida cotidiana. Resulta difícil esperar una transformación o recreación de los imaginarios geográficos de los usuarios en el curso de una revolución digital que invisibiliza las espacialidades digitales de la experiencia espacial del sujeto.

\section{Orientación pragmática en el diseño de la interfaz}

La pregunta que surge es qué tipo de desarrollos en la interfaz conducen a este renacimiento de conceptos espaciales tradicionales en lugar de conceptos como el cuerpo del individuo, topónimos coloquiales para la ciudad o la nación definida como una comunidad espacial.

La historia de la tecnología en general, y de las tecnologías digitales en particular, señala que el progreso tecnológico ha conducido a una nueva libertad en el diseño de las interfaces. Las restricciones tecnológicas han ido disminuyendo: el proceso de diseño ha dejado de estar determinado desde la caja negra de la estructura y los diseñadores vienen experimentando más posibilidades de explorar nuevas oportunidades en las últimas décadas. El vocabulario evidencia este cambio con el pasaje desde un lenguaje de máquinas a un lenguaje gráfico o simbólico, con variaciones de acuerdo a la filosofía de diseño de cada interfaz (Hartmann, 2012).

Con simultaneidad a esta mayor libertad tecnológica, ha tomado más fuerza el criterio de interfaces amigables para los usuarios: el diseño se basa en la simplicidad para el usuario y en la incorporación de la lógica de la vida cotidiana en las interfaces (Hellinge, 2008). Así, las guías contemporáneas de diseño de interfaces sugieren la adaptación del diseño al conocimiento que tiene un usuario lego. Esto significa que el lenguaje verbal y visual de las interfaces debe aproximarse al imaginario pre-existente en lugar de desafiarlo. En relación a la representación espacial el diseño de interfaces es, entonces, inherentemente conservador. Las ideas tradicionales de espacio y lugar son re-introducidas en las interfaces de diseño con conceptos como paisaje, región, lugar, nación.

\section{Conclusión}

Presentamos aquí un modelo descriptivo de la interacción con la tecnología e infraestructura digital en las prácticas cotidianas que asume tres formas de interacción: numérica/ algorítmica, deíctica y toponímica que, de modo general, expresan el sistema tecnológico, el usuario individual y la comunidad. Cada una de estas formas de interacción comprende y considera el espacio de modo diferente. En efecto, la interacción con la tecnología digital podría comprenderse como la permanente (re)-producción de geografías simbólicas muy diferenciadas y las prácticas de interacción, como una traducción constante e imbricada de cada una de estas categorías. Cuando interactuamos con las tecnologías digitales cambiamos permanentemente los modos de hacer el lugar y el espacio. El análisis de algunos casos empíricos a partir de este modelo indica que las categorías deícticas y los topónimos se han tornado cada vez más relevantes en los lenguajes interactivos. 
Tilo FELgENHAUER

La revolución digital, paradójicamente, ha privilegiado conceptos como lugar, localidad, región, nación en el diseño de las interfaces en lugar de reconfigurar las geografías cotidianas en términos numéricos y algorítmicos. Por un lado, los usuarios se alejan cada vez más de los complejos mecanismos y procesos que se esconden detrás de la superficie de las interfaces y solo reciben los efectos de estas tecnologías en sus vidas cotidianas. De este modo, las tecnologías no cuestionan los imaginarios geográficos de los usuarios. Por otro lado, el principio de lograr interfaces amigables promueve una adaptación al conocimiento medio de los usuarios desde el propio diseño, en vez de desafiarlas. El diseño de interfaces toma y se integra a los imaginarios geográficos y así, contribuye a reforzarlos. Probablemente esto explique porque todavía nos resulta más familiar el lugar- mundo que un mundo de imaginaciones globales y utópicas.

Traducción al español de Verónica Hollman.

El autor agradece a los dos evaluadores anónimos por sus comentarios y a Verónica Hollman por la traducción del artículo. 


\section{Q Bibliografía}

》 Abend, P. (2013). The Uses of Geomedia: An Object-Centered and Situated Approach. En: T. Jekel, A. Car, J. Strobl y G. Gerald (Eds.), GI_Forum 2013. Creating the GISociety (pp. 338-348). Berlín: Wichmann.

》 Ash, J. y Simpson, P. (2014). Geography and post-phenomenology. Progress in Human Geography, 1-19. DOI 10.1177/0309132514544806

》 Bialasiewicz, L. (2003). Another Europe: remembering Habsburg Galicja. Cultural Geographies, 10(1), 21-44.

» Berger, P. y Luckmann, T. (1966). The social construction of reality. A treatise in the sociology of knowledge. Nueva York: Anchor Books.

》 Bittner, C., Glasze, G. y Turk, C. (2013). Tracing contingencies: analyzing the political in assemblages of web 2.0 cartographies. GeoJournal, 78(2), 935-948.

» Blake, M., Mellor, J. y Crane, L. (2010). Buying Local Food: Shopping Practices, Place, and Consumption Networks in Defining Food as "Local". Annals of the Association of American Geographers, 100(2), 409-426.

» Blumenberg, H. (1981). Wirklichkeiten in denen wir leben. Stuttgart: Reclam.

» Bollnow, O.F. (2010 [1963]). Mensch und Raum. Stuttgart: Kohlhammer.

"Bradley, D. J. (1988). The scope of travel medicine. An introduction to the Conférence on Inteernational Travel Medicine. En: H., DuPont y R., Steffen (Eds.), Travel Medicine and health. (pp.1-19). Berlín: Springer.

》 Bromley, R. (2010). Storying community: Re-imagining regional identities through public cultural activity. European Journal of Cultural Studies, 13(1), 9-25.

»Buliung, R. (2011). Wired People in Wired Places: Stories about Machines and the Geography of Activity. Annals of the Association of American Geographers, 101(6), 1365-1381.

„ Buttimer, A. (1976). Grasping the dynamism of lifeworld. Annals of the Association of American Geographers, 66(2), 277-292.

"Cairncross, F. (1997). The Death of Distance. How the Communications Revolution Is Changing Our Lives. Boston: Harvard Business School Press.

》 Castells, M. (2000). Materials for an exploratory theory of the Network society, British Journal of Sociology, 51(1), 1-24.

»De Certau, M. (1984). The practice of everyday life. Berkeley/Los Ángeles: University of California Press.

" Dicken, P. (1998). Global Shift. Transforming the world economy. London/ Thousand Oaks/New Dehli: Sage.

»Dodge, M. y Kitchin, R. (2005). Code and the Transduction of Space. Annals of the Association of American Geographers, 95(1), 162-180.

»Edensor, T. (2002). National Identity, Popular Culture and Everyday Life. Oxford/ Nueva York: Berg. 
》 Eisel, U; Schultz, H.-D. (2008). Klassische Geographie. Geschlossenes Paradigma oder variabler Denkstil? Berliner Geographische Arbeiten, 111. Berlín: Geographisches Institut.

»Elwood, S., Schuurman, N., Wilson, M. (2011). Critical GIS. En: T. Nyerges, H. Couclesis y R. McMaster. The SAGE Handbook of GIS and Society (pp. 87-106). Los Angeles: Sage.

»Felgenhauer, T., Mihm, M. y Schlottmann, A. (2005). The making of 'Mitteldeutschland' On the function of implicit and explicit symbolic features for implementing regions and regional identity. Geografiska Annaler, Series $B, 87(1)$, 45-60.

" Gaspari, A.C. (1799). Lehrbuch der Erdbeschreibung zur Erläuterung des neuen methodischen Schul-Atlasses. Weimar: Erster Cursus.

" Gehlen, A. (1958). Der Mensch: seine Natur und seine Stellung in der Welt. Bonn: Athenäum.

» Gehlen, A. (2007 [1957]). Die Seele im technischen Zeitalter. Frankfurt: Klostermann.

» Giddens, A. (1984). The Constitution of Society. Cambridge: Polity.

" Giddens, A. (1990). Consequences of Modernity. Stanford: Stanford University Press.

" Goodchild, M. (2007). Citizens as sensors: the world of volunteered geography. GeoJournal, 69(4), 211-221.

"Graham, S. (1998). The End of geography or the explosion of space? Conceptualizing space, place and information technology. Progress in Human Geography, 22(2), 165-185.

» Graham, S. (2005). Software-sorted geographies. Progress in Human Geography, 29(5), 562-580.

》 Gregory, D. (1994). Geographical Imaginations. Cambridge: Blackwell.

» Gryl, Inga y Jekel, T. (2012). Re-centering geoinformation in secondary education: Toward a spatial citizenship approach. Cartographica: The International Journal for Geographic Information and Geovisualization, 1(47), 18-28.

» Günzel, S. (2010). Medialer Raum: Bilder - Zeichen - Cyberspace. En: S. Günzel (Ed.). Raum. Ein interdisziplinäres Handbuch (pp. 219-233) Stuttgart: Metzler.

" Harraway, D. (2013). Simians, Cyborgs, and Women: The Reinvention of Nature. Londres: Routledge.

》 Hartmann, F. (2012). Symbiosis yet? Koevolution der Grenzfläche Mensch/ Maschine. En: G. Freyermuth y L. Gotto (Eds.). Bildwerte. Visualität in der digitalen Medienkultur (pp. 259-285). Bielefeld: Transcript.

» Hellige, H. (2008). Krisen- und Innovationsphasen in der Mensch-ComputerInteraktion. En: H. Hellige (Ed.). Mensch-Computer-Interface. Zur Geschichte und Zukunft der Computer-Bedienung (pp. 11-92). Bielefeld: Transcript.

» Herder, J. (2001 [1772]). Abhandlung über den Ursprung der Sprache. Stuttgart: Reclam.

» Kingsbury, P. y Jones, J. (2009). Walter Benjamin's Dionysian Adventures on Google Earth. Geoforum, 40(4), 502-513. 
" Kitchin, R. y Dodge, M. (2011). Code/space: software and everyday life. Cambridge: The MIT Press.

» Kwan, M. (2007). Affecting Geospatial Technologies: Towards a Feminist Politics of Emotion. The Professional Geographer, 59(1), 22-34.

》Latour, B. y November, V. y Camacho-Hübner, E. (2010). Entering a risky territory. Space in the age of digital navigation. Environment and Planning D: Society and Space, 28(4), 581-599.

" Livingstore, D. (2008 [1992]). The Geographical Tradition. Episodes in the History of a Contested Enterprise. Malden/Oxford/Carlton: Blackwell.

》 Lois, C. y Hollman, V. (2013). Geografía y cultura visual. Los usos de las imágenes en las reflexiones sobre el espacio. Rosario: Prohistoria Ediciones.

》 Lough, B. y Moore McBride, A. (2014). Navigating the boundaries of active global citizenship. Transactions of the Institute of British Geographers, 39(3), 457-469.

»Mann, S. (2001). Cyborg: Digital Destiny and Human Possibility in the Age of the Wearable Computer. Nueva York: Random House.

» Marshall, T. (2014). Prisoners of Geography. Ten Maps that explain Everything about the World. Londres: Elliott \& Thompson.

» McLuhan, M. (1964). Understanding media: the extensions of man. Nueva York: McGraw-Hill.

》 Mersch, D. (2006). Medientheorien zur Einführung. Hamburg: Junius.

» Miller, V. (2011). Understanding Digital Culture. Los Angeles: Sage.

"Paasi, A. (2003). Region and place: regional identity in question, Progress in Human Geography, 27(4), 475-485.

»Perkins, C. (2014). Plotting practices and politics: (im)mutable narratives in OpenStreetMap, Transactions of the Institute of British Geographers, 39, 304-317.

》Pinker, S. y Goldstein, R. (2012). The long reach of reason. TED conference talk. Recuperado de http://www.ted.com/talks/steven_pinker_and_rebecca_ newberger_goldstein_the_long_reach_of_reason?language=en

» Ritter, C. (1822). Die Erdkunde im Verhältniß zur Natur und zur Geschichte des Menschen, oder allgemeine vergleichende Geographie, als sichere Grundlage des Studiums und Unterrichts in physicalischen und historischen Wissenschaften. Berlin: Reimer.

" Robertson, R. (1995). Glocalization: Time-Space and Homogeneity-Heterogeneity. En: M. Featherstone, S. Lash y R. Robertson (Eds.). Global Modernities (pp. 25-44). Londres/Thousand Oaks/New Dehli: Sage.

» Sauer, C. (1963 [1925]). The Morphology of Landcape. Berkeley: Berkeley University Press.

» Schultz, H.-D. (1998). Deutsches Land -deutsches Volk. Die Nation als geographisches Konstrukt. En: Berichte zur deutschen Landeskunde (72), 2, 85114.

» Schütz, A.y Luckmann, T. (2003). Strukturen der Lebenswelt. Konstanz: UVK, 7181.

»Semple, E. (1911). Influences of Geographic Environment on the Basis of Ratzel's 
TILO FELgenHAUER

System of Anthropo-Geography. Nueva York: Holt.

"Shaw, I. y Warf, B. (2009). Worlds of affect: virtual geographies of video games. Environment and Planning A, 41(6), 1332-1343.

»Simpson, P. (2014). Spaces of Affect. En: P. Adams, J. Craine y J. Dittmer (Eds.). The Ashgate Research Companion to Media Geography (pp. 329-345). Farnham/ Burlington: Ashgate.

"Sui, D. (2004). GIS, Cartography, and the "Third Culture": Geographic Imaginations in the Computer Age. The Professional Geographer, 56(1), 62-72.

" Thompson, J. (1995). The Media and Modernity. A Social Theory of the Media. Stanford: Stanford University Press.

»Thrift, N. (1996). Spatial Formations. London: Sage.

»Thrift, N. (2004a). Remembering the technological unconscious by foregrounding knowledges of position. Environment and Planning D: Society and Space, 22(1), 175-190.

»Thrift, N. (2004b). Intensities of Feeling: Towards a Spatial Politics of Affect. Geografiska Annaler, Series B, 86(1), 57-78.

» Turner, F.J. (1996). The Frontier in American History. Nueva York: Dover.

»Virilio, P. (1999). Fluchtgeschwindigkeit. Frankfurt: Fisher.

"Wellman, B. (2002). Little Boxes, Glocalization, and Networked Individualism. En: M. Tanabe, P. van den Besselaar y T. Ishida (Eds.). Digital Cities, LNCS (pp. 10-25). Berlin/Heidelberg: Springer.

"Wodak, R., de Cillia, R., Reisigl, M. y Liebhart, K. (2009). The Discursive Construction of National Identity. Edimburgo: Edinburgh University Press.

Tilo Felgenhauer / tilo.felgenhauer@uni-jena.de

Tilo Felgenhauer es profesor asistente en el Departamento de Geografía Social de la Universidad de Jena, Alemania. Con formación en geografía, historia y ciencia política su principal área de investigación es la construcción lingüística y visual de las regiones y de las identidades regionales, particularmente en relación con los medios de comunicación. Actualmente estudia cómo las prácticas cotidianas (como la movilidad, la comunicación y el consumo) dependen de la interacción con interfases digitales. 